\title{
Fabrication of Cylindrical Lenses by Combining Ultrashort Pulsed Laser and $\mathrm{CO}_{2}$ Laser
}

\author{
Simon Schwarz* and Ralf Hellmann* \\ *Applied Laser and Photonics Group, University of Applied Sciences, \\ 63743 Aschaffenburg, Germany \\ E-mail: Simon.Schwarz@h-ab.de
}

\begin{abstract}
We report on the fabrication of cylindrical lenses by combining an ultrashort pulsed laser ablation process with a subsequent $\mathrm{CO}_{2}$ laser polishing step. Firstly, a $1030 \mathrm{~nm}$ ultrashort pulsed laser is used to ablate a designed geometry in fused silica in a layer-by-layer process. In a comprehensive parameter study, we find that the ablation height depends exponentially on the pulse-to-pulse distance and linear on both laser fluence and layer repetitions. This facilitates to precisely choose the step height of the multi-layer ablation process to about $1 \mu \mathrm{m}$, which allows maintaining a high contour accuracy for fabricating and shaping 3D objects. In a subsequent step, a defocused $10.6 \mu \mathrm{m}$ $\mathrm{CO}_{2}$ laser is applied to solely polish the rough surface of the 3D objects, i.e. the $\mathrm{CO}_{2}$ laser does not shape the finally targeted object but rather fuses and thereby smoothens the surface. Microscopic measurements reveal an improvement of the surface roughness of about $0.6 \mu \mathrm{m}$ after the ablation process by two orders of magnitude after the $\mathrm{CO}_{2}$ laser smoothing process. The functionality of thus fabricated cylindrical lenses is tested using a HeNe laser. A typical beam profile for cylindrical lenses, which remarkably corresponds to the intensity profile of a Gaussian beam, is observed. This photonic process chain paves the way towards an all optical 3D micro-shaping technology with a high degree of geometrical freedom for medium sized volume production to single component rapid prototyping.
\end{abstract}

DOI: 10.2961/jlmn.2017.02.0005

Keywords: ultrashort pulsed laser, $\mathrm{CO}_{2}$ laser, laser ablation, laser polishing, fused silica, optic fabrication

\section{Introduction}

Micro-optical elements are widely used in numerous applications such as, e.g., optical communication, microopto-electro-mechanical systems, lab-on-a-chip devices and sensor applications [1-3]. Various microsystem technology based approaches are available for the fabrication of micro-optics on an industrial scale [4-6]. Recently, laser based processes such as laser direct writing $[3,7,8]$ or a combination of laser and etching [1] as well as a combination of different lasers [2, 9] have also been reported. In particular, ultrashort pulsed lasers have gained interest in processing transparent materials. During the irradiation by means of the high intensity ultrashort pulsed laser, electrons are excited from the valance to the conduction band due to a) multi-photon absorption and b) avalanche ionization, which are further heated by the incident laser radiation, before the energy is transferred from the excited electrons to the lattice $[10,11]$. This abrupt energy transfer causes ablation of the material without any heat transfer to the surrounding material, offering great advantage for contactless and thus gentle and wear-free optical fabrication with freely selectable geometry.

Bliedtner et al. [12] used an ultrashort pulsed laser to generate optical freeforms in different glass substrates, yet without generating optical quality surfaces. Pan et al. [1] fabricated micro-lens arrays using an ultrashort pulsed laser with a subsequent wet etching process. Choi et al. [2] combined a femtosecond and a $\mathrm{CO}_{2}$ laser to create micro optics and highlight its functionality by demonstrating micro lens arrays. In a first step, the ultrashort pulsed laser generates geometrical preforms that are subsequently transformed into the desired optical component geometry. The required curvatures are introduced to the preforms by a focussed $\mathrm{CO}_{2}$ laser induced melting, which is accompanied by a surface polishing. Contrary to this approach, Heidrich et al. [9] used a $\mathrm{CO}_{2}$ laser to generate and polish the optical compound in a two-step process and, finally, employ an ultrashort pulsed laser in a third step to correct minor form deviations.

In this contribution, we expand these all laser based approaches by differently employing the ultrashort pulsed laser for sole geometry definition and a defocussed $\mathrm{CO}_{2}$ laser for subsequent sole polishing. We exemplify the potential of this new approach, by fabricating cylindrical lenses and prove their optical imaging function by measuring a Helium-Neon laser with a CCD camera.

Opposite to Ref. 2, our new approach offers a higher degree of freedom in the generation of complex geometries and leads to a remarkably lower surface roughness. In addition, using a defocussed $\mathrm{CO}_{2}$ laser for polishing features the potential of a higher process efficiency. As compared to Ref. 9, the ability for tighter focussing of the ultrashort pulsed laser for the ablation step as compared to the $\mathrm{CO}_{2}$ laser offers the possibility to generate smaller structures with higher contour accuracy. Moreover, a third process step for form correction is not required, i.e. by reducing the number of process step again process efficiency is increased. 


\section{Experiment}

Two laser systems form the basis of the proposed new all optical 3D micro-shaping technology. An ultrashort pulsed laser initially forms the desired 3D geometry by laser ablation, whereas a $\mathrm{CO}_{2}$ laser subsequently fuses and thereby smoothens the generated surface. The Yb:KGW ultrashort pulsed laser (Pharos, Light Conversion) has a pulse duration of 222 fs (FWHM), an emission wavelength of $1030 \mathrm{~nm}$, and a repetition rate of $50 \mathrm{kHz}$. Combined with a Galvo scanner (RTA AR800 2G+, Newson) mounted on an adjustable z-stage, the laser spot is scanned across the specimens surface. The $\mathrm{f}-\theta$-lens with a focal length of $100 \mathrm{~mm}$ leads to a focal beam diameter of $31 \mu \mathrm{m}\left(1 / \mathrm{e}^{2}\right)$ as being measured with a high resolution CCD camera (UI-1490SEM-GL, IDS). For the ablation process, a scanning strategy of hatched layers is used. For the generation of the 3D objects, the z-axis is adjusted after each layer to maintain the focal position on the respective surface.

The $\mathrm{CO}_{2}$ laser (Infinity, Iradion) is specified by an output power of $60 \mathrm{~W}$ and a beam diameter of $5 \mathrm{~mm}\left(1 / \mathrm{e}^{2}\right)$ measured with a beam profiler (FocusMonitor, Primes), before focusing with a lens having a focal length of about $80 \mathrm{~mm}$. Yet, for the smoothening step the $\mathrm{CO}_{2}$ laser is defocussed to ensure a flattish and homogeneous intensity across a larger area of ablated lines. The scanning strategy is also a hatch of parallel lines, realized by flying optics.

To exemplarily demonstrate the potential of the proposed 3D micro-shaping approach, polished fused silica (GVB solutions in glass) is chosen due to its wide-ranging usage for optical components [13, 14]. A laser scanning microscope (LSM VK-X200, Keyence) is used to analyse the generated $3 \mathrm{D}$ structures and characterize the surface roughness.

\section{Results and Discussion}

\subsection{Ultrashort pulsed laser ablation}

To get acquainted with the ablation process and to determine the achievable ablation step height, we perform a comprehensive parameter study varying the applied laser fluence, pulse-to-pulse distance during scanning and the number of ablated layers, respectively. To ensure a comparable surface morphology during these experiments and the layer wise structuring process, particularly for the first ablated layer, we initially roughen the polished fused silica specimen. For this roughening, a fluence of $2.38 \mathrm{~J} / \mathrm{cm}^{2}$ and a pulse-to-pulse distance of $4 \mu \mathrm{m}$ are used in both $\mathrm{x}$ and $\mathrm{y}$ direction, leading to a surface roughness of $R_{a}=0.63 \mu \mathrm{m}$. Please note, this roughening step does not contribute to the subsequent 3D structuring process and can be omitted if unpolished glasses are structured.

Figure 1a) shows the influence of the pulse-to-pulse distance on the ablated step height (generated after the aforementioned pre-roughening), while the fluence is kept to $2.38 \mathrm{~J} / \mathrm{cm}^{2}$. For this variation, the scanning speed and the line distance of the hatch was varied while the laser repetition rate remains constant. Apparently, the step height decreases exponentially with increasing pulse-to-pulse distance from $25.06 \mu \mathrm{m}$ to $0.59 \mu \mathrm{m}$. A similar behavior has previously been reported by Bliedtner et al. during ablation of borosilicate glass [12]. A variation of the applied fluence between $1.75 \mathrm{~J} / \mathrm{cm}^{2}$ and $4.83 \mathrm{~J} / \mathrm{cm}^{2}$ (pulse-to-pulse distance of $4 \mu \mathrm{m}$ ) reveals a linear increase of the ablated step height between $5.97 \mu \mathrm{m}$ and $15.12 \mu \mathrm{m}$ with higher fluence leading to larger step heights (Fig. 1b)).

In order to generate surfaces with the ultrashort pulsed laser close to the desired geometry with high surface accuracy and smoothest transitions between adjacent ablation steps, we select a small step height of about $1 \mu \mathrm{m}$ per ablation layer. This can be achieved by either using low fluence or high pulse-to-pulse distance. To guarantee high process efficiency, a higher pulse-to-pulse distance is preferable. As a compromise of accuracy and process time, a fluence of $2.38 \mathrm{~J} / \mathrm{cm}^{2}$ and a pulse-to-pulse distance of $12 \mu \mathrm{m}$ are chosen, accordingly resulting in a step height of about $1.16 \mu \mathrm{m}$ (Fig. 1a)).

To generate an optical component with a total height of $300 \mu \mathrm{m}$ requires the ablation of about 300 such layers. In order to maintain a stable ablation process with constant step heights throughout all layers, we have investigated the step height increase with the number of layer repetitions for the selected parameter combination of laser fluence $2.38 \mathrm{~J} / \mathrm{cm}^{2}$ and pulse-to-pulse distance of $12 \mu \mathrm{m}$. Figure 1c) depicts the increase of the step height for up to 20 layer repetitions. Also the accumulated step height of the layerwise material removal increases linearly with the number of layers with an average step height of $0.96 \mu \mathrm{m}$ per layer (gradient of figure 1c)). Thus, the 3D micro-shaping approach can be well controlled by the number of repetitions. Such a linear dependence of the ablation depth allowed also the precise structuring of silicon carbide [15], aluminum nitride and alumina [16].

To demonstrate the abilities of the ablation process, a

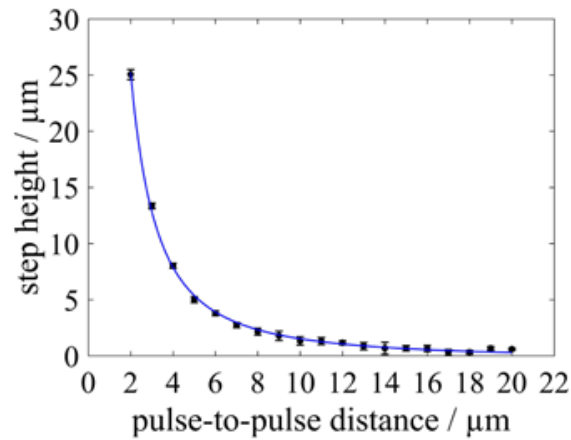

a)

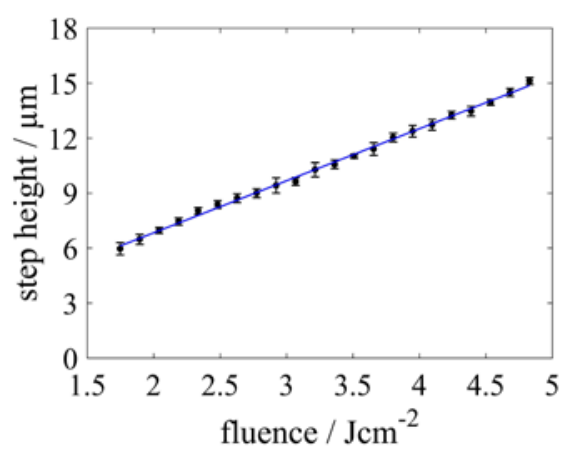

b)

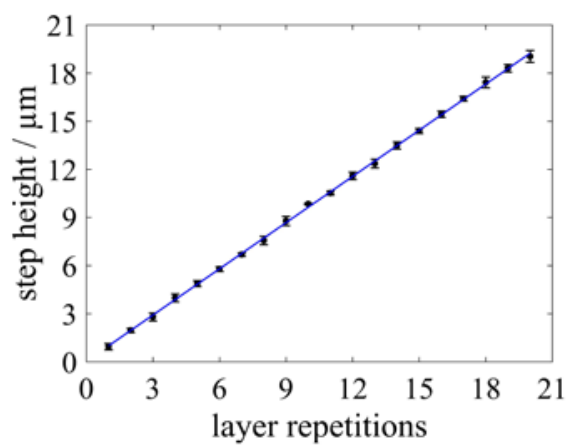

c)

Fig. 1 Parameter evaluation for the ultrashort pulsed laser ablation process, step height depending on a) pulse-to-pulse distance, b) fluence and c) layer repetitions (errors bars include the standard deviation of five LSM step heights measurements). 


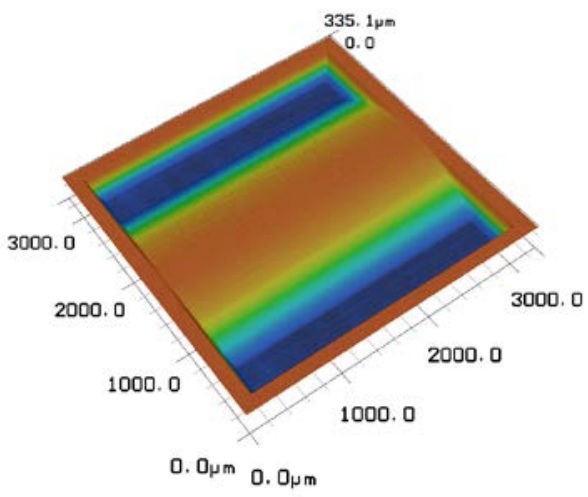

a)

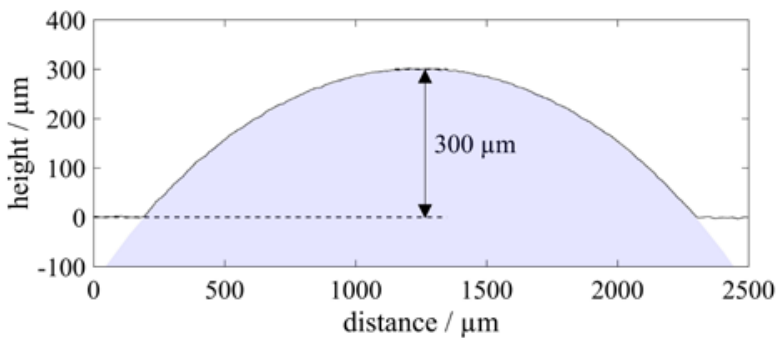

b)

Fig. 2 Generated 3D object a) LSM image and b) crosssection comparing measured (black line) and regular circle having a radius of $2 \mathrm{~mm}$ (gray blue region).

cylindrical lens having a radius of $2 \mathrm{~mm}$ and a height of $300 \mu \mathrm{m}$ is generated. For this purpose a negative of the lens is constructed with a CAD program and sliced into individual layers having a layer-to-layer distance of $0.96 \mu \mathrm{m}$. Figure 2a) depicts a LSM image of the thus generated component using the afore determined parameters (pulse-to-pulse distance $12 \mu \mathrm{m}$ and fluence $2.38 \mathrm{~J} / \mathrm{cm}^{2}$ ), representing the geometric form of a cylindrical lens with an excellent agreement of the achieved component height of $300 \mu \mathrm{m}$ and overall curvature. This is highlighted in figure 2b), comparing a cross section of the generated cylindrical lens (LSM measurement, black line in Fig. 2b)) and a drawn circle with a radius of $2 \mathrm{~mm}$.

The surface roughness after the entire ablation step is determined on top of the cylindrical lens to $R_{a}=0.63 \mu \mathrm{m}$, which is about a factor of 7 superior to the roughness reported after femtosecond laser fabrication in Ref. 2. However, even this low $R_{a}$ does not meet the surface requirements for optical components and excludes optical functionality. Consequently, a following polishing step is mandatory.

The high shape accuracy while the layer-by-layer ablation process with step heights of about $1 \mu \mathrm{m}$ offers the possibility for medium sized volume production with flat but also spherical geometries. Even complex shapes like freeform surfaces for optical use can be manufactured with this 3D micro-shaping process, overcoming conventional processing methods.

\section{2 $\mathrm{CO}_{2}$ laser polishing}

In order to achieve optical surface quality, a $\mathrm{CO}_{2}$ laser is used to polish the afore fabricated fused silica cylindrical lens. According to Ref. 9 and 17, we employed defocused laser polishing, which has been proven to be preferential for achieving smooth fused silica surfaces. By analyzing the effects of defocusing distance (difference between nominal focal length and set distance between focusing optics and specimen), scanning speed and laser power, we optimize the contour accuracy and surface roughness. As a result, we find for our machine setup a preferential process parameter set of $60 \mathrm{~W}$ laser power modulated with $13.3 \mathrm{~Hz}$, $20.3 \mathrm{~mm} / \mathrm{min}$ translation speed, and a hatch distance of $250 \mu \mathrm{m}$, respectively.

Figure 3 compares cross-sections of thus polished and unpolished cylindrical lenses for a distances of defocusing $\mathrm{d}_{\mathrm{f}}$ of $127 \mathrm{~mm}$ (red line) and $\mathrm{d}_{\mathrm{f}}$ of $142 \mathrm{~mm}$ (blue line). The resulting spot diameters increase to $8 \mathrm{~mm}\left(1 / \mathrm{e}^{2}\right)$ for the $127 \mathrm{~mm}$ defocusing and $9 \mathrm{~mm}\left(1 / \mathrm{e}^{2}\right)$ for the $142 \mathrm{~mm}$ defocusing. As a result of the laser polishing the overall height of the fabricated lens is reduced by about $5 \mu \mathrm{m}$, which we attribute to a partial evaporation of the material by excess energy deposition beyond the liquefaction. Nonetheless, the surface roughness is significantly reduced to, e.g., $\mathrm{R}_{\mathrm{a}}=6 \mathrm{~nm}$ at the top of the lens for $\mathrm{d}_{\mathrm{f}}=127 \mathrm{~mm}$.

In terms of contour accuracy, it appears that the ablated sharp edge at the rim of the unpolished cylindrical lens (cf. left inset in Fig. 3) is flattened. Such a rounding during laser smoothing has also been observed by Wlodarczyk et al. [18], due to the transfer of the molten viscous layer from the top to the bottom of the structure. Against this background, the previously described reduced height can not be attributed to this behaviour because for $d_{f}=142 \mathrm{~mm}$, no rounding is observed, while the total height is also reduced. In general, we find that this effect can be avoided by a further defocusing, yet with the drawback of a higher surface roughness, in turn limiting applicability in optics. For example, for $d_{f}=142 \mathrm{~mm}$ we maintain the original ablated contour (blue line in Fig. 3), however with $R_{a}$ increasing to $54 \mathrm{~nm}$ at the top of the lens.

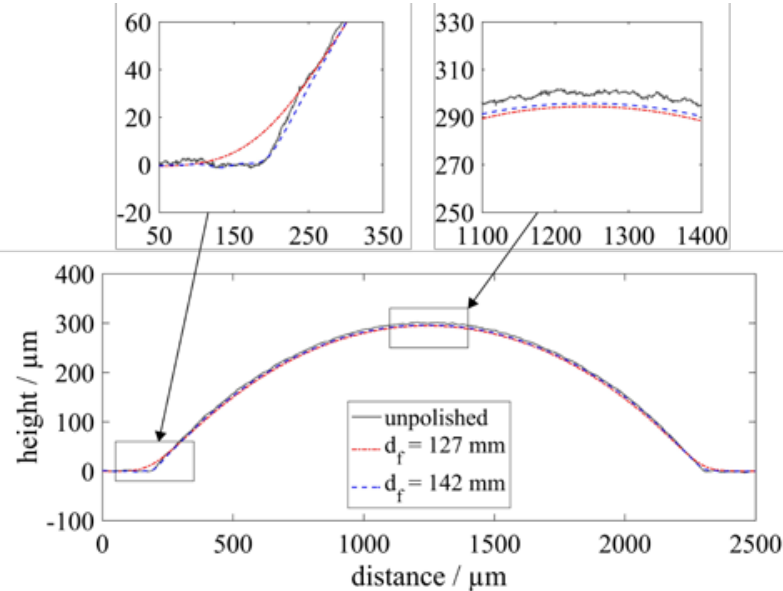

Fig. 3 LSFM cross-sections of generated 3D structures of an unpolished and two polished cylindrical lenses, enlargements highlight the form deviation.

\subsection{Testing the fabricated lenses}

To assess the functionality and quality of the finished optical component, a HeNe laser (HRR005S, Thorlabs) having a wavelength of $632.8 \mathrm{~nm}$ is imaged by the cylin- 
drical lens with the beam profile being measured with a CCD camera (BC106N-VIS/M, Thorlabs). Figure 4 compares the beam profile of the HeNe laser without the lens a) and the resulting profile measured beyond the focal length of the cylindrical lens b) at the same position in the optical pass. We highlight the excellent imaging properties, namely the good consistency with the Gaussian beam profiles (red lines in $\mathrm{x}$ - and $\mathrm{y}$-direction) and the homogeneity in the elliptically shaped beam profile of the fabricated optical component. The diameter of the HeNe laser at the entrance of the cylindrical lens measured to be about $725 \mu \mathrm{m}\left(1 / \mathrm{e}^{2}\right)$.

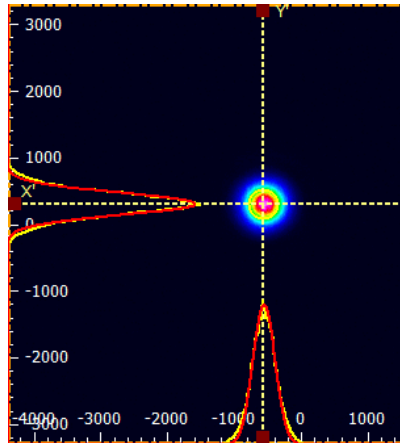

a)

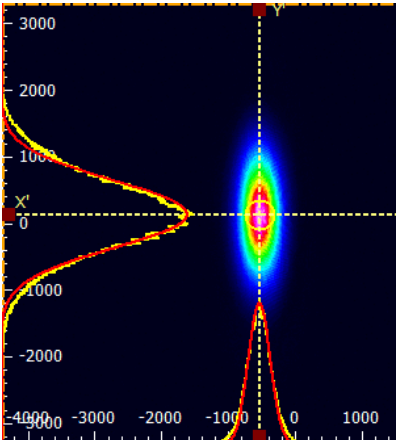

b)
Fig. 4 CCD camera image of HeNe laser beam profile a) without and b) with the cylindrical lens fabricated with $\mathrm{d}_{\mathrm{f}}=127 \mathrm{~mm}$ during polishing (red lines are Gaussian fits to the measured data).

\section{Conclusion}

We have demonstrated and optimized a new all laser based 3D micro-shaping process chain being capable to fabricate freeform optical components. Combining femtosecond laser ablation for basic geometry definition and subsequent $\mathrm{CO}_{2}$ laser polishing high quality optical components can be realized. The potential of this approach is exemplarily highlighted by generating a fused silica cylindrical lens with a high degree of contour accuracy after the ultrashort pulsed laser ablation process and a surface roughness as low as $6 \mathrm{~nm}$ after the subsequent polishing process. The functionality of the optical component is demonstrated by its basic imaging properties. Due to its high degree of geometrical freedom, form accuracy and optical quality, this 3D micro-shaping process chain has potentials for both individualized rapid prototyping of single components and midsized volume production.

\section{References}

[1] A. Pan, B. Gao, T. Chen, J. Si, C. Li, F. Chen, and X. Hou: Opt. Express 22 (2014), 15245

[2] H. K. Choi, J. Ryu, C. Kim, Y. C. Noh, I. B. Sohn, and J. T. Kim: J. Laser Micro Nanoengin. 11 (2016), 341

[3] C. H. Lin, L. Jiang, Y. H. Chai, H. Xiao, S. J. Chen, and H.-L. Tsai: Appl. Phys. A 97 (2009), 751

[4] M. B. Stern, and T. R. Jay: Opt. Eng. 33 (1994), 3547

[5] P. Nussbaum, R. Völkel, H. P. Herzig, M. Eisner, and S. Haselbeck: Pure Appl. Opt. 6 (1997), 617

[6] D. L. MacFarlane, V. Narayan, J. A. Tatum, W. R. Cox, T. Chen, and D. J. Hayes: IEEE Photon. Technol. Lett. 6 (1994), 1112

[7] Y. L. Zhang, Q. D. Chen, H. Xia, and H. B. Sun: Nano Today 5 (2010), 435
[8] D. Nieto, J. Arines, C. Gomez-Reino, G. M. O’Connor, and M. T. Flores-Arias: J. Appl. Phys. 110 (2011), 023108

[9] S. Heidrich, E. Willenborg, and A. Richmann: Phys. Proc. 12 (2011), 519

[10]B. C. Stuart, M. D. Feit, A. M. Rubenchik, B. W. Shore, and M. D. Perry: Phys. Rev. Lett. 74 (1995), 2248

[11]M. Lenzner, J. Krüger, S. Sartania, Z. Cheng, C. Spielmann, G. Mourou, W. Kautek, and F. Krausz: Phys. Rev. Lett. 18 (1998), 4076

[12] J. Bliedtner, C. Schindler, M. Seiler, S. Wächter, M. Friedrich, and J. Giesecke: LTJ 5 (2016), 46

[13]H. Bach, and N. Neuroth: The Properties of Optical Glass, Springer Science \& Business Media (2012)

[14] M. J. Weber: Handbook of Optical Materials, CRC Press (2002)

[15]B. Adelmann, A. Hürner, G. L. Roth and R. Hellmann: J. Laser Micro Nanoengin. 10 (2015), 190

[16]F. Preusch, B. Adelmann and R. Hellmann: Micromachines 5 (2014), 1051

[17]C. Weingarten, A. Schmickler, E. Willenborg, K. Wissenbach, and R. Poprawe: J. of Laser App. 29 (2017), 011702

[18] K. L. Wlodarczyk, E. Mendez, H. J. Baker, R. McBride, and D. R. Hall: Appl. Opt. 49 (2010), 1997

(Received: April 3, 2017, Accepted: June 27, 2017) 